\title{
Clinical Trials of Probiotic Strains in Selected Disease Entities
}

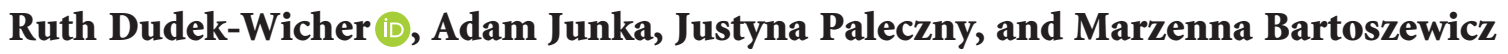 \\ Department of Pharmaceutical Microbiology and Parasitology, Faculty of Pharmacy, Medical University of Silesian Piasts, \\ Wrocław, Poland \\ Correspondence should be addressed to Ruth Dudek-Wicher; r.dudek.wicher@gmail.com
}

Received 15 April 2020; Accepted 18 May 2020; Published 28 May 2020

Academic Editor: Joseph Falkinham

Copyright (C) 2020 Ruth Dudek-Wicher et al. This is an open access article distributed under the Creative Commons Attribution License, which permits unrestricted use, distribution, and reproduction in any medium, provided the original work is properly cited.

\begin{abstract}
Probiotics are live microorganisms which when administered in adequate amounts confer a health benefit on the host. Although their mechanism of action is not clearly explained, it is known that they positively modulate the immune system, which leads to immunity potentiation. A number of studies prove that probiotics strengthen cognitive functions, reduce anxiety, and regulate the lipid metabolism in the human body. Probiotics used in humans are most often of the Lactobacillus and Bifidobacterium species. However, as more research is conducted, new species with beneficial, probiotic properties are being discovered. This paper provides a review of available information about the influence of probiotics on human health. It summarizes the current knowledge on the mechanism of action of probiotics as well as clinical trial results proving their efficacy in allergic, neurodegenerative, and cardiac diseases. This review also discusses the data concerning the safety of probiotics in clinical treatment.
\end{abstract}

\section{Introduction}

For many years, the development of research has been aimed at characterizing the human intestinal microbiome and determining the role of individual species present in it. Probiotic strains (probiotics) are defined as "live microorganisms which when administered in adequate amounts confer a health benefit on the host" [1]. When ingested or applied to the skin, probiotics interact with the microbiome that inhabits the respective niches of the body. Currently, increased nutritional awareness of consumers in developed societies is observed. The above translates into consumer interest in food products that can not only satisfy hunger but also fulfill additional physiological and nutritional functions, by improving health or preventing diseases. The main advantage of probiotics is their impact on the development of the microbiome in a way that ensures a proper balance between pathogens and bacteria necessary for the proper functioning of the body.

For this reason, probiotics are widely used to restore the normal composition of the microbiome after antibiotic therapy. There are also reports of the special role of probiotics in the prevention and treatment of obesity, diabetes, allergies, asthma, lung diseases, autoimmune diseases, HIV (Human Immunodeficiency Virus) infections, cancers, urogenital infections, and gastrointestinal diseases such as diarrhea, irritable bowel syndrome, necrotizing enterocolitis, or cirrhosis, as well as in the eradication of Helicobacter pylori infections. The reader can find these studies in an extensive review by Hill et al. [1].

This article discusses the probable mechanism of action of probiotics and highlights their use in the prevention and treatment of selected disease entities.

\section{Probiotics}

Probiotic properties are associated with specific strains of the microorganisms. In order for a strain to be described as "probiotic," it has to meet a number of requirements related to safety, functionality, and technological suitability $[2,3]$. The safety profile is determined based on the strain's origin, degree of antibiotic resistance, and no relationship to pathogenic strains. When assessing functionality, the ability to survive and maintain metabolic activity and growth at the target site is taken into account, as well as antagonistic activity against pathogens such as Helicobacter pylori, 
Salmonella spp., Listeria monocytogenes, or Clostridium difficile. Technological usefulness is demonstrated, for example, by the effective production of large amounts of biomass and high productivity of cultures and their resistance to bacteriophages [3]. Probiotic microorganisms used in humans mainly belong to the Lactobacillus and Bifidobacterium species. Not only are they free from lipopolysaccharides that cause inflammation, but they also release active molecules that help keep the intestines and skin healthy. Other commonly used probiotics are Lactococcus, Streptococcus, and Enterococcus, as well as Bacillus clausii, Enterococcus faecium SF68, and some yeast strains of the genus Saccharomyces. The Escherichia coli Nissle 1917 strain is a unique probiotic which synthesizes the semirough lipopolysaccharide (LPS) and does not produce P- and S-fimbrial adhesins, which are important virulence factors in other E. coli strains. Due to these features, E. coli Nissle 1917 has no pathogenic effect and can be used in the treatment of gastrointestinal diseases [4].

A list of the most commonly used probiotic strains contained in pharmaceutical products and used as food additives is presented in Table 1.

\section{Mechanisms of Action of Probiotics}

The mechanism by which probiotics interact with the host is most likely pleiotropic. The strongest clinical evidence of the beneficial effect of probiotics on human health is their immunomodulatory activity manifested in an increase in body immunity $[7,8]$. The intestines, together with the gutassociated lymphoid tissue (GALT), play an important role in ensuring and maintaining the body's immunity. Imbalances in this system lead to inflammation in the digestive system. It is believed that increased mucosal permeability and loss of intestinal epithelial cells integrity (IECs) also play a role in the pathogenesis of other diseases [6-8].

It has been shown that probiotics affect every part of the intestinal tissue (such as the mucus barrier, epithelium, lamina propria), vessels and nerves of its components, smooth muscles that control intestinal peristalsis, and mesenteric lymph nodes that have the ability to communicate with the immune system. One of the possible mechanisms of action is a direct improvement of the intestinal defense barrier by reducing mucosal permeability and improving epithelial integrity and the interaction of probiotics with commensal organisms [7]. Probiotics play an important role in regulating both the innate and adaptive immune system by activating macrophages, NK cells, and cytotoxic T cells, by modulating IgA production, stimulating toll-like receptors (TLRs), and modifying the cytokine expression profile $[7,8]$. The beneficial effect of commensal bacteria, e.g., lactobacilli, is associated with the stimulation of pattern-recognition receptors (PRRs), especially TLRs, on the surface of enterocytes and dendritic cells. These receptors recognize microbial-associated molecular patterns (MAMPs), and their activation triggers numerous cascades for intracellular transmission, which in turn leads to the production of endogenous antibacterial agents, defensins, proinflammatory cytokines, and chemokines. Thanks to the above, the mechanisms are activated conditioning the tightness of the intestinal barrier and limiting the presence of microorganisms in the intestinal lumen. Through these mechanisms, probiotics facilitate the creation of the immune system of a newborn child, among others, by balancing the activity of helper lymphocyte subpopulations Th1 and Th2 and stimulating regulatory $\mathrm{T}$ cell (Treg) subpopulations [9]. It has also been reported that probiotics can produce metabolites with anti-inflammatory local and systemic activity and, as a consequence, indirectly affect the inflammatory reaction. These metabolites are mainly short-chain fatty acids (SCFAs) such as butyric, acetic, and propionic acid $[9,10]$. SCFAs bind to G-protein-coupled receptors to induce intracellular signaling. Therefore, SCFAs act as mediators between the microbiome and the brain, thanks to which intestinal bacteria influence the physiology and behavior of the brain [11]. They activate the receptors (FFAR2, FFAR3, or GPR109A) on the intestinal epithelial cells, which leads to an inhibition of nuclear secretion of NF- $\kappa \mathrm{B}$ transcription factor by B lymphocytes. SCFAs also have the ability to inhibit histone deacetylases in Treg lymphocytes, which increases their number and consequently suppresses an excessive immune response. SCFAs also stimulate the ileal and colon L cells to produce gastrointestinal hormones such as pancreatic polypeptide YY (PYY) and glucagon-like peptide-1 (GLP-1). In 1993, it was demonstrated for the first time that intravenous administration of the PYY peptide reduced appetite. The main task of GLP-1 is to stimulate insulin secretion, and the receptors for this peptide are found in the digestive system - the intestine and the endocrine part of the pancreas-and in the central nervous system (CNS) [12]. SCFAs stimulate tolerogenic dendritic cells (DCs) that trigger CD4 + T cells to differentiate into Tregs. These effects inhibit the production of cytokines by neutrophils and macrophages by interacting with the receptors. The tryptophan and indole derivatives produced by probiotics interact with the AhR (aryl hydrocarbon receptor) while adenosine and the inosine derivative interact with the adenosine-2A receptor. Both receptors are located on $\mathrm{T}$ lymphocytes, and the interactions of the above compounds cause an anti-inflammatory effect. Histamine produced by some probiotic strains interacts with the $\mathrm{H} 2$ receptor present on intestinal epithelial cells and macrophages. This interaction results in a decrease in the level of proinflammatory cytokines (TNF- $\alpha, \mathrm{MCP}-1$, and IL-12) [10].

Bacteria have the ability to produce and release neurotransmitters such as $\gamma$-aminobutyric acid (GABA), serotonin, catecholamines, and histamine or their precursors. Neurotransmitters send signals to the CNS through enterochromaffin cells and intestinal nerve receptors. GABA, the main inhibitory neurotransmitter in the CNS, whose dysfunction is associated with depression, anxiety, autism, and schizophrenia, is produced in human intestines by Lactobacillus brevis and Bifidobacterium dentium [11]. Other products of probiotic metabolism are bacteriocins, which can be compared to an antibiotic. They include acidoline, acidophylline, lactacin, lactocidin, reuterin, lactoline, and enterocin. Still other metabolites exhibit anticancer or 
TABLe 1: Exemplary probiotic strains used in humans $[2,5,6]$.

\begin{tabular}{|c|c|c|c|}
\hline Genera Lactobacillus & Genera Bifidobacterium & Genera Streptococcus & Other \\
\hline $\begin{array}{l}\text { L. acidophilus } \\
\text { L. amylovorus } \\
\text { L. casei } \\
\text { L. delbrueckii } \\
\text { L. fermentum } \\
\text { L. gasseri } \\
\text { L. helveticus } \\
\text { L. johnsonii } \\
\text { L. pentosus } \\
\text { L. plantarum } \\
\text { L. reuteri } \\
\text { L. rhamnosus }\end{array}$ & $\begin{array}{l}\text { B. adolescentis } \\
\text { B. animalis } \\
\text { B. bifidum } \\
\text { B. breve } \\
\text { B. infantis } \\
\text { B. longum }\end{array}$ & $\begin{array}{l}\text { S. thermophilus } \\
\text { S. lactis } \\
\text { S. intermedius } \\
\text { S. salivarius } \\
\text { S. cremoris }\end{array}$ & $\begin{array}{c}\text { Saccharomyces boulardii } \\
\text { Escherichia coli Nissle } 1917 \\
\text { Enterococcus faecium SF68 } \\
\text { Bacillus clausii } \\
\text { Lactococcus lactis } \\
\text { Propionibacterium freudenreichii } \\
\text { Pediococcus acidilactici }\end{array}$ \\
\hline
\end{tabular}

immunosuppressive activity. The antimicrobial properties of probiotics include not only the production of antimicrobial compounds, but also competition with pathogens for adhesion to the epithelium and to nutrients. The ability of probiotic strains to coaggregate enables the formation of a protective barrier preventing the colonization of the epithelium by pathogens. In addition, they have the ability to inhibit the production of bacterial toxins. Probiotics have been proven to increase the synthesis and absorption of vitamins (mainly from group $\mathrm{B}$, but also $\mathrm{PP}$ and $\mathrm{K}$ ) and mineral compounds and to stimulate the production of organic acids and amino acids. They may also be able to produce mucus as well as enzymes such as esterase, lipase, and coenzymes A, Q, NAD, and NADP $[2,13]$. Probiotics have been shown to effectively lower total cholesterol and low-density lipoproteins (LDL). There are several proposed mechanisms of action of probiotics on total cholesterol and LDL levels. These are enzymatic deconjugation of bile acids by hydrolysis of bile salts, ability to bind cholesterol in the small intestine, assimilation and incorporation of cholesterol into cell membranes of probiotics, conversion of cholesterol into coprostanol, or reduction of cholesterol esters in LDL particles [13].

It can also be assumed that probiotic strains may be responsible for the detection and degradation of potential carcinogens [13, 14]. Lactic acid bacteria present in the intestine play a role in carcinogenesis regression because of their effect on immunomodulation. In addition, they have the ability to both increase and decrease the production of anti-inflammatory cytokines, which play an important role in preventing carcinogenicity. They are also able to activate phagocytes to eliminate cancer cells at an early stage. The use of probiotic bacteria killed with high temperature in combination with radiation helped increase the detection of cancer cells. A reduced resistance to carcinogens has been observed in germ-free mice. There are many cohort studies showing a correlation between the consumption of dairy products and the risk of colon and colorectal cancer [14].

\section{Allergic Diseases}

Allergic diseases have become a serious health concern in recent decades. The number of cases of atopic dermatitis, food allergies, or asthma is constantly increasing, especially in Western societies. To explain this phenomenon, the "hygiene hypothesis" is often invoked, which claims that a reduced exposure to microbes in early life leads to imbalances between Th1/Th2 lymphocytes. This hypothesis is confirmed by studies of the GABRIEL group ("A Multidisciplinary Study to Identify the Genetic and Environmental Causes of Asthma in the European Community," GABRIEL, Advanced Study), which clearly indicate that the risk of atopy or asthma was significantly lower in children who had contact with environmental microorganisms compared to children raised in cities [15]. Numerous studies were conducted to explain the complicated mechanism of allergy development [16-23]. In a clinical trial it was observed that L. rhamnosus GG (LGG) reduces the concentration of exhaled nitric oxide in 4-7 year olds with asthma. However, the use of Lactobacillus reuteri, Lactobacillus rhamnosus HN001, Lactobacillus paracasei subsp. paracasei F19, Bifidobacterium bifidum, B. lactis, and Lactococcus lactis in infants did not reduce asthma symptoms [17]. More favorable results were obtained by examining the effectiveness of probiotics in the prevention and treatment of eczema and atopic dermatitis. Kukkonen et al. proved that giving pregnant women complex probiotics (Lactobacillus rhamnosus, Bifidobacterium breve, and Propionibacterium freudenreichii) significantly reduces the risk of atopic dermatitis in children up to the age of two [18]. The preventive effect of LGG has also been demonstrated in another clinical study conducted on pregnant women in New Zealand [19]. A meta-analysis of 21 clinical trials in which prenatal and postnatal women were given probiotics showed that they are effective in preventing but not treating atopic dermatitis [20]. The results of another meta-analysis showed the preventive effect of probiotics on the development of eczema, but the effectiveness of the use of probiotics for other allergic diseases has not been confirmed. However, the effect is moderate, and the only probiotic strain with reproducible data is Lactobacillus rhamnosus GG (LGG). The World Allergy Organization (WAO) suggests the use of probiotics in pregnant and lactating women and infants only if there is a high risk of hereditary allergies [21].

The dramatic increase in the prevalence and severity of food allergies around the world is also prompting the search for new therapeutic strategies. Dysbiosis of intestinal microbiome early in life is a critical factor in the 
development of food allergies. The intestinal microbiome is therefore a promising goal of innovative therapeutic and prophylactic strategies. Evidence confirms that probiotics modulate immune tolerance by affecting the structure and function of intestinal microbiota, interacting with enterocytes, and changing nonimmune mechanisms such as intestinal permeability and mucus thickness and through immunogenic mechanisms including the stimulation of sIgA and $\beta$-defensin production, as well as modulation of cytokine response by immune cells. A beneficial effect in alleviating food allergies has been proven for L. plantarum and $B$. adolescentis and for the probiotic mixture $L$. casei W56, L. lactis W58, L. acidophilus W55, L. salivarius W57, B. infantis W52, B. lactis W18, and B. longum W51 [22]. Neau et al. confirmed the preventive effect on food allergy to cow's milk of 3 new strains of Lactobacillus salivarius LA307, Bifidobacterium longum subsp. infantis LA308, and Lactobacillus rhamnosus LA305 [23]. In a clinical study conducted on 220 children with confirmed allergy to cow's milk, it was shown that the administration of highly hydrolyzed casein in combination with the strain Lactobacillus rhamnosus GG reduced the occurrence of allergic symptoms and accelerated the acquisition of cow's milk protein tolerance [24].

Peanut allergy is the second most common allergic condition, especially in children. Australian researchers conducted a study that used probiotic and peanut oral immunotherapy (PPOIT). The therapy lasted for 18 months, and $L$. rhamnosus CGMCC1.3724 was used as a probiotic. At the end of the study, it was observed that $89.7 \%$ of the participants in the PPOIT group were desensitized compared to $7.1 \%$ in the placebo group. In addition, the researchers checked the condition of the children participating in the study after 4 years. Their allergic reactions to nuts were checked, skin tests were performed, and the concentration of sIgE and sIgG4 antibodies to peanuts was measured. It was shown that $67 \%$ of children from the PPOIT group could safely eat peanuts. Although the results of the study are promising, further research is needed to confirm that PPOIT therapy may become a cure for peanut allergy $[25,26]$.

The results of the above-described studies indicate the high therapeutic potential of probiotics, although further, more detailed research is necessary to fully understand the potential of probiotics in the fight against allergic diseases.

\section{Heart and Circulatory System}

Cardiovascular disease (CVD) is a leading cause of death worldwide. There are numerous reports on the beneficial properties of certain probiotic strains used in treatments aimed at lowering cholesterol and treating hypertension. It has been proven that probiotics directly protect against strokes, by producing certain proteins and by activating heat shock proteins, and also that they prevent hypertrophy after a heart attack. In addition, they regulate fat metabolism, affecting the size of fat cells, and they regulate the circulation of leptin and adiponectin. It has also been found that proteins produced by LGG such as $\mathrm{p} 75$ have dose-dependent cardioprotective effects and reduce the risk of ischemic stroke. Similar properties were observed for proteins produced by L. plantarum 299v [27]. The cardioprotective effect of probiotics on the heart muscle has been proven in animal model studies. Inhibition of proinflammatory cytokine production and reduction of oxidative stress have also been exhibited by B. breve, L. casei, L. bulgaricus, and $L$. acidophilus. Therefore, probiotic supplements may find use as an additional prophylactic option in patients at risk of coronary heart disease $[28,29]$. High cholesterol, especially the LDL fraction, is a major precursor of hypertension, hyperlipidemia, and coronary heart disease and also causes plaque buildup in the arteries. The serum LDL fraction maintained within the optimal range reduces the chances of these diseases occurring. In a study in which a meta-analysis of randomized clinical trials was conducted on 1971 patients, it was shown that probiotic strains, i.e., $L$ acidophilus, $L$. Lactis, and L. plantarum, significantly reduce the level of total serum cholesterol [30]. Lowering the total cholesterol and LDL fraction in all groups compared to the control group was observed in a study involving 485 patients with high, borderline, and normal cholesterol [31]. In clinical trials, strains such as Lactobacillus plantarum ECGC 13110402, Lactobacillus fermentum ME-3, Bifidobacterium lactis HN019, Streptococcus thermophilus, Lactobacillus acidophilus L1, Bifidobacterium longum BL1, and Lactobacillus plantarum 299v showed a total cholesterol level lowering effect. The most favorable results were obtained in the case of L. acidophilus and L. plantarum [13]. To date, Health Canada has only approved one probiotic product recommended for the treatment of cardiovascular disease. The product-Cardioviva ${ }^{\mathrm{TM}}$, also available in the USA and Europe, contains 2 billion encapsulated Lactobacillus reuteri NCIMB 30242 bacteria, which have been clinically proven to lower LDL cholesterol by $11.6 \%$ in adults with hypercholesterolemia [32].

Hypertension is closely related to hypercholesterolemia. It has been proven that selected strains of the genera Lactobacillus and Bifidobacterium are effective in lowering blood pressure because they produce peptides that act similarly to drugs from the group of angiotensin converting enzyme (ACE) inhibitors [33]. A meta-analysis of 14 clinical trials with 702 participants showed that fermented probiotic milk significantly reduced both systolic and diastolic blood pressure in hypertensive patients. The increase in the population of Lactobacillus and Bifidobacterium caused by the administration of fermented milk caused a proportional increase in proteolytic activity and inhibition of ACE [34].

The available meta-analyses have some limitations, and hence more randomized multicenter studies should be conducted to broaden the knowledge about the possibility of using probiotics in the treatment of cardiovascular diseases.

\section{Neurodegenerative Diseases}

Numerous studies describe the effect of intestinal microbiome on human health and homeostasis. Moreover, research also describes the possible role of the microbiome in the pathogenesis of brain function disorders. Neuropsychiatric diseases have various causes. The emerging evidence of the interaction between the brain, intestines, and 
microbiome can help explain the mechanisms underlying these complex interactions. A two-way information exchange takes place on the gut-brain axis. Direct and indirect exchange mechanisms include nerve (vagus, intestinal nerves), hormonal (serotonin, monoamines, GABA, neutrophilic brain factor), and immune pathways. It is believed that changes in the intestinal microbiome are a possible cause of some brain diseases, including Parkinson's disease (PD), Alzheimer's disease (AD), and multiple sclerosis (MS). In studies conducted on adult patients, a reduction in the percentage of Prevotellaceae was found in the stool samples of patients with PD compared to the control group. A greater share of Enterobacteriaceae was observed in people with postural instability and movement difficulties. The composition of the microbiome was also studied in children with relapsing-remitting MS. A reduction in the number of Fusobacteria was significantly associated with the risk of recurrence. A decrease in the number of bacteria from the Prevotella and Lactobacillus genera has been observed in people with MS [10]. In the light of the above findings, it seems reasonable to test probiotics for their properties supporting the treatment of $\mathrm{PD}, \mathrm{AD}$, and MS diseases. $\alpha$-Synuclein plays an important role in the process of neurodegeneration. It is a protein abundant in neurons, whose physiological role is not fully understood. However, a wealth of data indicates its involvement in the release of neurotransmitters from synaptic endings and in neuronal plasticity. Normal functions of this protein are disturbed during its aggregation. Aggregated $\alpha$-synuclein is involved in cell death in neurodegenerative diseases, including Parkinson's disease, Alzheimer's disease with Lewy bodies, and dementia with Lewy bodies. $\alpha$-Synuclein activates TLR receptors, which leads to the formation of an inflammatory reaction preceding the degeneration of neurons. Therefore, inhibition of TLR receptors delays the progression of PD or $\mathrm{AD}$. The best known probiotic strains of the genera Lactobacillus and Bifidobacterium interact with TLRs, helping to reduce the inflammation. Therefore, they can be important in preventing the degeneration of neurons [35]. A study by Akbari et al. showed that a probiotic supplement consisting of Lactobacillus acidophilus, Lactobacillus casei, Bifidobacterium bifidum, and Lactobacillus fermentum administered for 3 months improves the cognitive functions of patients with $\mathrm{AD}$ [36]. The effectiveness of "psychobiotics" has also been confirmed in a study conducted on 20 patients with Alzheimer's disease. Markers of intestinal inflammation were examined and stool samples were analyzed before and after 4 weeks of supplementation with a probiotic consisting of Lactobacillus casei W56, Lactococcus lactis W19, Lactobacillus acidophilus W22, Bifidobacterium lactis W52, Lactobacillus paracasei W20, Lactobacillus plantarum B62, Lactobacillus plantarum W23, and Lactobacillus salivarius W24. After treatment, an increase in Faecalibacterium prausnitzii was observed compared to baseline. At the same time, kynurenine serum level has increased. The results indicate that, in patients with Alzheimer's disease, supplementation with a multispecies probiotic affects the composition of intestinal bacteria, suggesting that microbiome modulation may affect the development and course of
Alzheimer's disease [37]. However, different results were obtained in a study of 60 patients aged 65-90 years in a very advanced stage of Alzheimer's disease. They received mixtures of probiotic strains: either Lactobacillus fermentum, Lactobacillus plantarum, and Bifidobacterium lactis or Lactobacillus acidophilus, Bifidobacterium bifidum, and Bifidobacterium longum for 3 months. This study showed that patients were insensitive to treatment. The authors emphasize that negative test results may be due to the use of other bacterial cultures and the inclusion of patients at a very advanced stage of the disease [38].

In a clinical trial, Parkinson's patients were given a mixture of the following strains for 12 weeks: Lactobacillus acidophilus $(2 \times 109 \mathrm{CFU} / \mathrm{g})$, Lactobacillus reuteri $(2 \times 109 \mathrm{CFU} / \mathrm{g})$, Bifidobacterium bifidum $(2 \times 109 \mathrm{CFU} / \mathrm{g})$, and Lactobacillus fermentum $(2 \times 109 \mathrm{CFU} / \mathrm{g})$. Improved movement and parameters such as CRP level, glutathione concentration, and insulin sensitivity were observed in the examined group [39]. Another study showed that oral administration of the Lactobacillus casei Shirota (LcS) strain for 4 weeks improved the motor function and reduced spasticity in the limbs. Furthermore, administration of the same strain led to a significant reduction in anxiety symptoms compared to the control group in patients with chronic fatigue syndrome. In addition, the administration of the same strain reduced abdominal pain, reduced flatulence, and improved stool consistency in PD patients. A beneficial effect, relieving the symptoms of Parkinson's disease, was also observed after the administration of L. helveticus R0052, B. longum R0175, B. animalis subsp. lactis, S. thermophiles, L. bulgaricus, and L. lactis subsp. lactis [40, 41]. One of the latest review articles discusses microbiological therapy as a novel treatment for Parkinson's disease. This analysis also contains data on the effects of stool transplantation in patients with Parkinson's disease [42].

It has been observed that MS patients have increased gene expression associated with proinflammatory immune pathways, including interferon, TLR, IL-6, and dendritic cell maturation. In these patients, a larger population of the Akkermansia and Methanobrevibacter species was also noted, compared to the number of these bacteria in the healthy control group. Akkermansia is a mucus-degrading bacterium, which in turn disturbs the functioning of the intestinal barrier, so there may be a direct relationship between the composition of the microbiota and the occurrence of MS [43]. Patients with MS also have a reduced amount of Lactobacillus bacteria. The administration of these probiotic strains resulted in increased bacterial diversity in the microbiome. Thus, the use of probiotics reduced the dysbiosis characteristic of MS and reduced the number of bacteria of the genera Akkermansia and Blautia. At the immune level, the administration of probiotics induced an anti-inflammatory peripheral immune response. In addition, in the control group with the MS risk gene (HLA-DQA1), a reduced expression of this gene was observed. These results suggest that probiotic treatment may have a synergistic effect [44]. There is also a study suggesting that $L$. reuteri alleviates the symptoms and improves the quality of life of patients with multiple sclerosis [10]. 
The potential usefulness of probiotics in the prevention or treatment of neurodegenerative diseases is becoming a widely studied topic. However, further research is needed to optimize the composition and dosage of simple and complex formulations and to develop optimal therapeutic regimens for individual neurodegenerative diseases. In addition, future research should also take into account the tolerability and safety of probiotics in patients with neurodegenerative diseases, as well as hormonal, immunological, neurochemical, and metabolic changes induced by probiotics or prebiotics. Therefore, further investment in large-scale clinical trials is needed to prove the efficacy of probiotics in neurodegenerative diseases [45].

\section{Probiotics Safety}

The American Food and Drug Administration (FDA) describes probiotics as Generally Recognized as Safe (GRAS). Based on the analysis of randomized clinical trials, potential applications of probiotics include the treatment of cystic fibrosis (e.g., Lactobacillus GG), autism spectrum disorders (e.g., L. plantarum WCFS1), Alzheimer's disease (e.g., L. acidophilus, L. casei, B. bifidum, L. fermentum), and celiac disease (e.g., B. infantis NLS, B. longum CECT 7347, B. breve BR03, B. breve B632); prevention of caries (e.g., Lactobacillus $G G)$, pneumonia associated with mechanical ventilation (e.g., L. casei rhamnosus, L. plantarum 299, B. longum $+L$. bulgaricus + Str. thermophilus), and urinary tract infections (e.g., Lactobacillus GG); and prevention of radiation-related symptoms (e.g., L. casei DN-114001). However, there are situations in which probiotics should not be administered. Such situations include immunosuppressive treatment, anticancer treatment, and acute pancreatitis. Caution is advised in the use of probiotics in premature babies, in patients with immunodeficiency, in patients with a catheter inserted into large veins, or in patients with a severe clinical condition. The administration of probiotics by jejunostomy may also be a risk factor. Cases of sepsis have also been reported in children with short bowel syndrome receiving LGG supplementation. In addition, several dozen cases of fungemia have been reported in individuals receiving $S$. boulardii as well as bacteremia in individuals receiving bacterial probiotics. In rare cases, probiotic bacteria may contain antibiotic resistance genes that they can pass on to other strains of bacteria, including harmful strains that cause infections $[46,47]$. Although probiotics are widely used in the treatment of postantibiotic diarrhea and Clostridium difficile infections, there is a lack of unambiguous data confirming the legitimacy of such therapy [48, 49].

\section{Summary}

Knowledge about the human intestinal ecosystem and its role in the maintenance of health is still limited. The mechanism of action of probiotic strains remains to be fully explained. Modulation of the microbiome composition and the effect of probiotics on the human body occurs through mechanisms such as bacteriocin and SCFA production, epithelial cell barrier modulation, innate immunity modulation (dendritic cell maturation), effect on Th1 to Th2 ratio, increase in the number and activity of regulatory T cells, and degradation of carcinogenic compounds [9-14].

A thorough understanding of these mechanisms is necessary to make full and safe use of the potential benefits that probiotic strains may bring to patients suffering from allergic, neurodegenerative, or cardiological diseases. The effectiveness of probiotics may vary depending on treatment, disease, and strain or strains that make up the probiotic. In addition, a probiotic may be effective in treating the disease, but not in its prevention. Research to date suggests that probiotics have beneficial and multifaceted effects on human health, which encourages further experimental and clinical research. Some probiotic activities are well documented, and their use alone or in combination with other therapies can be considered "evidence-based." However, in many cases further research is needed because the available evidence is insufficient to demonstrate the effectiveness of the probiotics themselves. Carefully designed multicenter clinical trials are needed to confirm the performance of individual probiotic strains administered at specific doses and to clarify the duration of therapy. Considering the contraindications to the use of probiotics and possible side effects, further research is needed on their safety.

\section{Data Availability}

The data supporting the conclusions of this paper are available through the articles cited in the reference list.

\section{Conflicts of Interest}

The authors declare that they have no conflicts of interest.

\section{Acknowledgments}

This work was supported by Medical University Statutory Funding SUB.D230.20.002.

\section{References}

[1] C. Hill, F. Guarner, G. Reid et al., "The international scientific association for probiotics and prebiotics consensus statement on the scope and appropriate use of the term probiotic," Nature Reviews Gastroenterology \& Hepatology, vol. 11, no. 8, pp. 506-514, 2014.

[2] P. Markowiak and K. Śliżewska, "Effects of probiotics, prebiotics and synbiotics on human health," Nutrients, vol. 9, no. 9, p. 1021, 2017.

[3] Food and Agriculture Organization (FAO), "Guidelines for the evaluation of probiotics in food," Report of a Joint FAO/ WHO Working Group on Drafting Guidelines for the Evaluation of Probiotics in Food, FAO, London, UK, 2002.

[4] F. Scaldaferri, V. Gerardi, F. Mangiola et al., "Role and mechanisms of action of Escherichia coli Nissle 1917 in the maintenance of remission in ulcerative colitis patients: an update," World Journal of Gastroenterology, vol. 22, no. 24, pp. 5505-5511, 2016.

[5] J. C. Sniffen, L. V. McFarland, C. T. Evans, and E. J. C. Goldstein, "Choosing an appropriate probiotic 
product for your patient: an evidence-based practical guide," PLoS One, vol. 13, no. 12, 2018.

[6] N. M. Meybodi and A. M. Mortazavian, "Probiotic supplements and food products: a comparative approach," Biochemistry \& Pharmacology: Open Access, vol. 6, no. 2, 2017.

[7] J. Plaza-Diaz, C. Gomez-Llorente, L. Fontana, and A. Gil, "Modulation of immunity and inflammatory gene expression in the gut, in inflammatory diseases of the gut and in the liver by probiotics," World Journal of Gastroenterology, vol. 20, no. 42, Article ID 15632, 2014.

[8] P. A. Bron, M. Kleerebezem, R.-J. Brummer et al., "Can probiotics modulate human disease by impacting intestinal barrier function?" British Journal of Nutrition, vol. 117, no. 1, pp. 93-107, 2017.

[9] A. Roży, P. Jaguś, and J. Chorostowska-Wynimko, "Rola probiotyków w profilaktyce i leczeniu chorób alergicznych," Pneumonologia i Alergologia Polska, vol. 80, no. 1, pp. 65-76, 2012.

[10] Y. Liu, J. Alookaran, and J. Rhoads, "Probiotics in autoimmune and inflammatory disorders," Nutrients, vol. 10, no. 10, p. $1537,2018$.

[11] Y.-K. Kim and C. Shin, "The microbiota-gut-brain axis in neuropsychiatric disorders: pathophysiological mechanisms and novel treatments," Current Neuropharmacology, vol. 16, no. 5, pp. 559-573, 2018.

[12] A. D. Silva and S. R. Bloom, "Gut hormones and appetite control: a focus on PYY and GLP-1 as therapeutic targets in obesity," Gut and Liver, vol. 6, no. 1, pp. 10-20, 2012.

[13] Y. Nazir, S. A. Hussain, A. A. Hamid, and Y. Song, "Probiotics and their potential preventive and therapeutic role for cancer, high serum cholesterol, and allergic and HIV diseases," BioMed Research International, vol. 2018, Article ID 3428437, 17 pages, 2018.

[14] A. Górska, D. Przystupski, M. J. Niemczura, and J. Kulbacka, "Probiotic bacteria: a promising tool in cancer prevention and therapy," Current Microbiology, vol. 76, no. 8, pp. 939-949, 2019.

[15] G. Loss, S. Apprich, M. Waser et al., "The protective effect of farm milk consumption on childhood asthma and atopy: the GABRIELA study," Journal of Allergy and Clinical Immunology, vol. 128, no. 4, pp. 766-773, 2011.

[16] S. Okada, H. Kita, T. J. George, G. J. Gleich, and K. M. Leiferman, "Migration of eosinophils through basement membrane components in vitro: role of matrix metalloproteinase-9," American Journal of Respiratory Cell and Molecular Biology, vol. 17, no. 4, pp. 519-528, 1997.

[17] M. Mennini, L. Dahdah, M. C. Artesani, A. Fiocchi, and A. Martelli, "Probiotics in asthma and allergy prevention," Frontiers in Pediatrics, vol. 5, 2017.

[18] K. Kukkonen, E. Savilahti, T. Haahtela et al., "Probiotics and prebiotic galacto-oligosaccharides in the prevention of allergic diseases: a randomized, double-blind, placebo-controlled trial," Journal of Allergy and Clinical Immunology, vol. 119, no. 1, pp. 192-198, 2007.

[19] C. Barthow, K. Wickens, T. Stanley et al., "The probiotics in pregnancy study (PiP study): rationale and design of a doubleblind randomised controlled trial to improve maternal health during pregnancy and prevent infant eczema and allergy," BMC Pregnancy Childbirth, vol. 16, no. 1, p. 133, 2016.

[20] J. Lee, D. Seto, and L. Bielory, "Meta-analysis of clinical trials of probiotics for prevention and treatment of pediatric atopic dermatitis," Journal of Allergy and Clinical Immunology, vol. 121, no. 1, pp. 116-121, 2008.
[21] C. E. West, M. Dzidic, S. L. Prescott, and M. C. Jenmalm, "Bugging allergy; role of pre-, pro-and synbiotics in allergy prevention," Allergology International, vol. 66, no. 4, pp. 529-538, 2017.

[22] R. B. Canani, L. Paparo, R. Nocerino et al., "Gut microbiome as target for innovative strategies against food allergy," Frontiers in Immunology, vol. 10, 2019.

[23] E. Neau, J. Delannoy, C. Marion et al., "Three novel candidate probiotic strains with prophylactic properties in a murine model of cow's milk allergy," Applied and Environmental Microbiology, vol. 82, no. 6, pp. 1722-1733, 2016.

[24] R. B. Canani, M. Di Costanzo, G. Bedogni et al., "Extensively hydrolyzed casein formula containing Lactobacillus rhamnosus GG reduces the occurrence of other allergic manifestations in children with cow's milk allergy: 3-year randomized controlled trial," Journal of Allergy and Clinical Immunology, vol. 139, no. 6, pp. 1906-1913, 2017.

[25] M. L. K. Tang, A.-L. Ponsonby, F. Orsini et al., "Administration of a probiotic with peanut oral immunotherapy: a randomized trial," Journal of Allergy and Clinical Immunology, vol. 135, no. 3, pp. 737-744, 2015.

[26] K.-C. Hsiao, A.-L. Ponsonby, C. Axelrad et al., "Long-term clinical and immunological effects of probiotic and peanut oral immunotherapy after treatment cessation: 4-year followup of a randomised, double-blind, placebo-controlled trial," The Lancet Child \& Adolescent Health, vol. 1, no. 2, pp. 97105, 2017.

[27] G. Ettinger, K. MacDonald, G. Reid, and J. P. Burton, "The influence of the human microbiome and probiotics on cardiovascular health," Gut Microbes, vol. 5, no. 6, pp. 719-728, 2014.

[28] J. Sadeghzadeh, A. Vakili, H. R. Sameni, M. Shadnoush, A.-R. Bandegi, and M. Z. Khorasani, "The effect of oral consumption of probiotics in prevention of heart injury in a rat myocardial infarction model: a histopathological, hemodynamic and biochemical evaluation," Iranian Biomedical Journal, vol. 21, no. 3, pp. 174-181, 2017.

[29] A. Uchinaka, N. Azuma, H. Mizumoto et al., "Anti-inflammatory effects of heat-killed Lactobacillus plantarum L-137 on cardiac and adipose tissue in rats with metabolic syndrome," Scientific Reports, vol. 8, no. 1, p. 8156, 2018.

[30] L. Wang, M.-J. Guo, Q. Gao et al., "The effects of probiotics on total cholesterol," Medicine, vol. 97, no. 5, Article ID e9679, 2018.

[31] Z. Guo, X. M. Liu, Q. X. Zhang et al., "Influence of consumption of probiotics on the plasma lipid profile: a metaanalysis of randomised controlled trials," Nutrition, Metabolism and Cardiovascular Diseases, vol. 21, no. 11, pp. 844850, 2011.

[32] M. L. Jones, C. J. Martoni, and S. Prakash, "Cholesterol lowering and inhibition of sterol absorption by Lactobacillus reuteri NCIMB 30242: a randomized controlled trial," European Journal of Clinical Nutrition, vol. 66, no. 11, pp. 1234-1241, 2012.

[33] C. Gonzalez-Gonzalez, T. Gibson, and P. Jauregi, "Novel probiotic-fermented milk with angiotensin I-converting enzyme inhibitory peptides produced by Bifidobacterium bifidum MF 20/5," International Journal of Food Microbiology, vol. 167, no. 2, pp. 131-137, 2013.

[34] J.-Y. Dong, I. M. Y. Szeto, K. Makinen et al., "Effect of probiotic fermented milk on blood pressure: a meta-analysis of randomised controlled trials," British Journal of Nutrition, vol. 110, no. 7, pp. 1188-1194, 2013. 
[35] V. Caputi and M. Giron, "Microbiome-gut-brain axis and toll-like receptors in Parkinson's disease," International Journal of Molecular Sciences, vol. 19, no. 6, p. 1689, 2018.

[36] E. Akbari, A. Asemi, R. D. Kakhaki et al., "Effect of probiotic supplementation on cognitive function and metabolic status in Alzheimer's disease: a randomized, double-blind and controlled trial," Frontiers in Aging Neuroscience, vol. 8, p. 256, 2016.

[37] F. Leblhuber, K. Steiner, B. Schuetz, D. Fuchs, and J. M. Gostner, "Probiotic supplementation in patients with Alzheimer's dementia-an explorative intervention study," Current Alzheimer Research, vol. 15, no. 12, pp. 1106-1113, 2018.

[38] A. Agahi, G. A. Hamidi, R. Daneshvar et al., "Does severity of Alzheimer's disease contribute to its responsiveness to modifying gut microbiota? a double blind clinical trial," Frontiers in Neurology, vol. 9, 2018.

[39] O. R. Tamtaji, M. Taghizadeh, R. D. Kakhaki et al., "Clinical and metabolic response to probiotic administration in people with Parkinson's disease: a randomized, double-blind, placebo-controlled trial," Clinical Nutrition, vol. 38, no. 3, pp. 1031-1035, 2019.

[40] G. Umbrello and S. Esposito, "Microbiota and neurologic diseases: potential effects of probiotics," Journal of Translational Medicine, vol. 14, no. 1, p. 298, 2016.

[41] V. Caputi and M. Giron, "Microbiome-gut-brain axis and toll-like receptors in Parkinson's disease," International Journal of Molecular Sciences, vol. 19, no. 6, p. 1689, 2018.

[42] X. Fang, "Microbial treatment: the potential application for Parkinson's disease," Neurological Sciences, vol. 40, no. 1, pp. 51-58, 2019.

[43] L. M. Cox and H. L. Weiner, "Microbiota signaling pathways that influence neurologic disease," Neurotherapeutics, vol. 15, no. 1, pp. 135-145, 2018.

[44] S. K. Tankou, K. Regev, B. C. Healy et al., "A probiotic modulates the microbiome and immunity in multiple sclerosis," Annals of Neurology, vol. 83, no. 6, pp. 1147-1161, 2018.

[45] H. Wang, I.-S. Lee, C. Braun, and P. Enck, "Effect of probiotics on central nervous system functions in animals and humans: a systematic review," Journal of Neurogastroenterology and Motility, vol. 22, no. 4, pp. 589-605, 2016.

[46] H. Szajewska, "Probiotyki-aktualny stan wiedzy i zalecenia dla praktyki klinicznej," Medycyna Praktyczna, vol. 7-8, pp. 19-37, 2017.

[47] A. N. Kunz, J. M. Noel, and M. P. Fairchok, "Two cases of Lactobacillus bacteremia during probiotic treatment of short gut syndrome," Journal of Pediatric Gastroenterology and Nutrition, vol. 38, no. 4, pp. 457-458, 2004.

[48] A. S. Kalakuntla, G. Nalakonda, K. Nalakonda, V. C. Pidikiti, and S. A. Aasim, "Probiotics and clostridium difficile: a review of dysbiosis and the rehabilitation of gut microbiota," Cureus, vol. 11, no. 7, Article ID e5063, 2019.

[49] S. Blaabjerg, D. Artzi, and R. Aabenhus, "Probiotics for the prevention of antibiotic-associated diarrhea in outpatients-a systematic review and meta-analysis," Antibiotics, vol. 6, no. 4, p. $21,2017$. 